\title{
Actividad ovárica del tepezcuintle Agouti paca (Rodentia: Agoutidae) en cautiverio
}

\author{
Rubén C. Montes Pérez \& Elsy A. Cabrera Baz \\ Facultad de Medicina Veterinaria y Zootecnia, Universidad Autónoma de Yucatán. Apdo. 4-116. CP. 97100. Fax: 999 \\ 9423205. Mérida, Yucatán, México; mperez@tunku.uady.mx; elsycab10@hotmail.com
}

\author{
Recibido 01-VIII-2001. Corregido 07-V-2005. Aceptado 02-XII-2005.
}

\begin{abstract}
Ovarian activity of Agouti paca (Rodentia: Agoutidae) under captivity. The ovarian activity of Agouti paca was characterized by hormonal profiles and ovarian structures. Samples of blood were taken from eight females (seven adults and one juvenile) at the breeding grounds of the Facultad de Medicina Veterinaria y Zootecnia in Yucatán, México. Sampling lasted approximately two months and was done every three and six days. Blood was collected from anesthetized animals, and the levels of progesterone (P4) and $17 \beta$ estradiol (E2) were analized by radioimmunoassay technique. Macroscopic and microscopic analyses were carried out in ovaries of dead animals. The estrous cycle lasted $29 \pm 8.4$ days, levels of $1.61 \pm 0.65 \mathrm{ng} / \mathrm{ml}$ for P4 and $39 \pm 24 \mathrm{pg} / \mathrm{ml}$ for E2 were observed for a follicular phase, $6.18 \pm 3.70 \mathrm{ng} / \mathrm{ml}$ and $29 \pm 16 \mathrm{pg} / \mathrm{ml}$ for P4 and E2 respectively in the luteal phase. Statistically significant differences were found between phases for P4 but not for E2. The presence of extragonadal steroids with levels of P4 of $1.9 \pm 0.77 \mathrm{ng} / \mathrm{ml}$ and E2 of $22 \pm 17 \mathrm{pg} / \mathrm{ml}$ were observed, which are not produced by the effects of managing stress. The changes in the levels of $\mathrm{P} 4$ during the cycle are indicators of luteal activity, with the intersticial tissue acting probably as active steroids-producing gland.Follicular growth was observed during the entire cycle. Rev. Biol. Trop. 54 (3): 903-912. Epub 2006 Sept. 29.
\end{abstract}

Key words: Agouti paca, steroids, estrous cycle, progesterone, estradiol, ovarian activity, hystricomorph.

Los estudios en Agouti paca que describen el desempeño reproductivo en cautiverio, indican que es una especie que tiene ciclos estrales durante todo el año y que los principales indicadores reproductivos son: el periodo de gestación, de aproximadamente 114 días, con un intervalo de 95 a 157 días; el tamaño de camada, que fluctúa entre una y tres crías; y el intervalo entre partos cuyo promedio es de 187 días con variación entre 178 y 191 (Sanvicente 1995, Smythe y Brown de Guanti 1995). La información sobre la duración del ciclo estral es variable. Matamoros y Pashov (1984), registraron cinco tipos de ciclo, cuyos períodos se extienden desde 6.8 hasta 37 días; sin embargo, Fierro y Morales (1995), mencionan un promedio de 31.2 días con variación de 18 a 51 . Matamoros y Pashov (1984) informaron tres etapas para el ciclo: proestro, estro y posestro, mientras que Fierro y Morales (1995) registraron cuatro: proestro, estro, metaestro y diestro. Ambos trabajos utilizaron exclusivamente la citología vaginal para describir el ciclo estral. En estos trabajos se indica que es necesario efectuar estudios endocrinológicos para precisar la actividad reproductiva del $A$. paca, los que aportarían nueva información que permitiría utilizarlos como indicadores de la reproducción, y de esta manera se podría determinar la ciclicidad reproductiva de las hembras, la presencia de anestro fisiológico (como la ocurrencia de la gestación), o el anestro patológico (representado por la presencia de quistes ováricos), y con ello tomar medidas apropiadas para realizar un manejo eficiente y seleccionar las hembras reproductoras (Montes 1995).

Los estudios sobre la endocrinología de la reproducción del $A$. paca mencionan que los 
niveles basales de progesterona varían entre 0.18 y $1.11 \mathrm{ng} / \mathrm{ml}$ de plasma sanguíneo y los máximos entre 1.79 y $77.27 \mathrm{ng} / \mathrm{ml}$; respecto a $17 \beta$ estradiol los niveles basales fluctúan entre 0.0 y $13.64 \mathrm{pg} / \mathrm{ml}$ de plasma y, los más altos entre 23.3 y 155.84 pg/ml (Cabrera y Montes 1999). Mientras que Guimarâes et al. (1999) informan que los niveles sanguíneos de progesterona fluctúan entre 1.73 y $1.8 \mathrm{ng} / \mathrm{ml}$ durante el estro; de 0.53 a $4.03 \mathrm{ng} / \mathrm{ml}$ en metaestro; entre $1.5 \mathrm{y} 16.08 \mathrm{ng} / \mathrm{ml}$ en diestro y de $0.45 \mathrm{a}$ $3.23 \mathrm{ng} / \mathrm{ml}$ en proestro.

El presente trabajo planteó determinar la presencia de esteroides extragonadales, describir los perfiles hormonales de progesterona y $17 \beta$ estradiol plasmáticos a lo largo del ciclo y relacionar las estructuras ováricas con los niveles de progesterona y $17 \beta$ estradiol.

\section{MATERIALES Y MÉTODOS}

El estudio se efectuó en el estado de Yucatán, México, cuyo clima es cálido subhúmedo clasificación $\mathrm{Aw}_{0}$ (García 1988), con una temperatura media anual de $26.8^{\circ} \mathrm{C}$, la precipitación pluvial anual de $984 \mathrm{~mm}$ y la humedad relativa media anual del 80\% (Duch 1988). Se trabajó con un total de ocho hembras A. paca (siete adultas y una juvenil), con un peso promedio de 4.4 kg (mínimo de 2.3 y máximo de $6.2 \mathrm{~kg}$ ) y dos machos castrados, uno con peso de $6 \mathrm{~kg}$ y otro con $7.5 \mathrm{~kg}$, todos fueron identificados por medio de tatuaje y se mantuvieron en corrales de $9 \mathrm{~m}^{2}$, disponiendo de comedero, bebedero, madriguera y recipiente para excretas. Los ejemplares fueron alimentados diariamente con frutas locales (papaya, naranja, cabalaza) en cantidades de $1.5 \mathrm{~kg}$ por animal y recibieron agua para beber a libertad.

La recolección de sangre en cada animal se realizó en intervalos de tres y seis días durante un período de dos meses, por medio de punción cardiaca y yugular (2 a $3 \mathrm{ml} /$ muestra/animal), las muestras sanguíneas se depositaron en tubos vacutainer con anticoagulante (solución acuosa de EDTA al 10\%). Previamente, los ejemplares fueron sedados por medio de inyec- ción intramuscular, usando una combinación de hidrocloruro de ketamina (16 mg/kg de peso) y xilazina (3 $\mathrm{mg} / \mathrm{kg}$ de peso). Las muestras de sangre fueron centrifugadas a 3000 revoluciones/min durante 5 min para obtener plasma, el cual fue almacenado a $-15^{\circ} \mathrm{C}$. En el plasma descongelado se efectuó la medición de progesterona (P4) y $17 \beta$ estradiol (E2) por radioinmunoanálisis (RIA) en fase sólida. Se utilizaron dos estuches comerciales de RIA para estimar los niveles de P4 y E2 (ImmuChem Coated Tube, ICN Pharmaceuticals Inc., y Coat-ACount, Diagnostic Products Co., respectivamente), previamente validados con pruebas de paralelismo y recuperación (Anónimo 1984) en muestras plasmáticas de $A$. paca, que contenían cantidades conocidas de esteroides.

Los machos adultos castrados, fueron muestreados semanalmente durante dos meses; también se recolectaron dos muestras sanguíneas en los mismos animales con una diferencia de tiempo de $40 \mathrm{~min}$. Las muestras de plasma sanguíneo se procesaron con los estuches comerciales previamente descritos, para estimar los niveles de P4 y E2.

En las hembras que gestaron durante el estudio, el análisis de los perfiles hormonales se efectuó hasta el momento en que se infirió que ocurrió el reconocimiento de gestación, este acontecimiento se ubicó cuando los niveles de P4 se elevaron sustancialmente sobre los niveles esperados de actividad luteal. Simultáneamente a la toma sanguínea se determinó la etapa del ciclo estral por medio de citología vaginal, según lo informado por Fierro y Morales (1995).

Se realizaron observaciones macro y microscópicas del aparato reproductor femenino a partir de órganos post mortem de cinco hembras que murieron durante el muestreo, debido a paro cardiorespiratorio o hemorragia interna. De estos órganos se obtuvieron cortes histológicos previamente incluidos en parafina, teñidos con hematoxilina y eosina y, finalmente montados en laminillas. Posteriormente se realizaron mediciones microscópicas de las estructuras ováricas. Se utilizó la prueba de Tstudent para contrastar los niveles de P4 y E2 
entre las fases folicular y luteal y de KruskallWallis para comparar los niveles hormonales entre las fases de proestro, estro, metaestro y diestro (Downie y Heath 1986).

\section{RESULTADOS}

Se recolectaron en total 109 muestras de sangre. En los machos castrados se obtuvieron 16 muestras, en éstos se observaron variaciones individuales en los niveles de P4 y E2, también se encontraron fluctuaciones en los niveles de ambos esteroides entre los dos momentos de muestreo, es decir en el intervalo de $40 \mathrm{~min}$; sin embargo, en ningún caso se detectaron diferencias significativas (T-student, $\mathrm{p}>0.05$ ). El valor medio de $\mathrm{P} 4$ en animales castrados fue de $1.95 \pm 0.77 \mathrm{ng} / \mathrm{ml}$ y para E2 de $23 \pm 17 \mathrm{pg} / \mathrm{ml}$.
Se observaron cinco ciclos estrales en tres hembras, bajo diferentes esquemas de muestreo (Cuadro 1) y niveles variables de esteroides en las diferentes etapas del ciclo (Cuadro 2); sin embargo, no se encontraron diferencias significativas (Kruskal-Wallis, $\mathrm{p}>0.05$ ) en los niveles de P4 y E2 entre las cuatro etapas del ciclo estral. Los niveles de esteroides en la fase folicular fueron de $1.6 \pm 0.65 \mathrm{ng} / \mathrm{ml}$ y $39 \pm 24 \mathrm{pg} /$ $\mathrm{ml}$ para P4 y E2 respectivamente, en tanto que, para la fase luteal fueron para P4 de 6.2 \pm 3.7 $\mathrm{ng} / \mathrm{ml}$ y de $29 \pm 16 \mathrm{pg} / \mathrm{ml}$ para E2. Se encontraron diferencias ( $\mathrm{T}$-student, $\mathrm{p}<0.05$ ) entre la fase folicular y luteal para $\mathrm{P} 4$, pero no entre la fase folicular y luteal para E2. Los perfiles hormonales de las hembras 4, 6, 7 y 8 y su correspondiente etapa del ciclo determinada por citología vaginal se muestra en la Fig. 1.

Las hembras 2 (Fig. 2B) y 4 (Fig. 2D) presentaron una proporción de E2 superior al de

CUADRO 1

Duración del ciclo estral en Agouti paca en diferentes esquemas de muestreo sanguíneo

TABLE 1

Estral cycle duration in Agouti paca in different times of blood sampling

\begin{tabular}{clccc} 
No. Animal & \multicolumn{1}{c}{ Esquema de Muestreo } & $\begin{array}{c}\text { Ciclo } \\
\text { (días) }\end{array}$ & $\begin{array}{c}\text { Fase Folicular } \\
\text { (días) }\end{array}$ & $\begin{array}{c}\text { Fase Luteal } \\
\text { (días) }\end{array}$ \\
4 & Cada 6 días & 30 & 8 & 22 \\
6 & Cada 3 días & 33 & 6 & 27 \\
6 & Cada 6 días & 18 & 6 & 12 \\
6 & Cada 6 días & 24 & 6 & 18 \\
8 & Cada 6 días & 40 & 10 & 30
\end{tabular}

\section{CUADRO 2}

Niveles de hormonas esteroides en cada etapa del ciclo estral en Agouti paca $(n=5)$

TABLE 2

Hormonal steroid levels on each estral phase in Agouti paca's cycle $(n=5)$

\begin{tabular}{lcccc}
\multicolumn{1}{c}{ Hormona } & Proestro & Estro & Metaestro & Diestro \\
Progesterona $(\mathrm{ng} / \mathrm{ml})$ & $1.6 \pm 1.8^{\mathrm{A}}$ & $1.9 \pm 1.7^{\mathrm{A}}$ & $12.8 \pm 9.2^{\mathrm{A}}$ & $9.4 \pm 8.9^{\mathrm{A}}$ \\
Estradiol $(\mathrm{pg} / \mathrm{ml})$ & $40 \pm 40^{\mathrm{B}}$ & $21 \pm 13^{\mathrm{B}}$ & $27 \pm 9^{\mathrm{B}}$ & $28 \pm 17^{\mathrm{B}}$
\end{tabular}

Valores con igual literal en una misma fila no difieren estadísticamente (Kruskal-Wallis, p>0.05). 

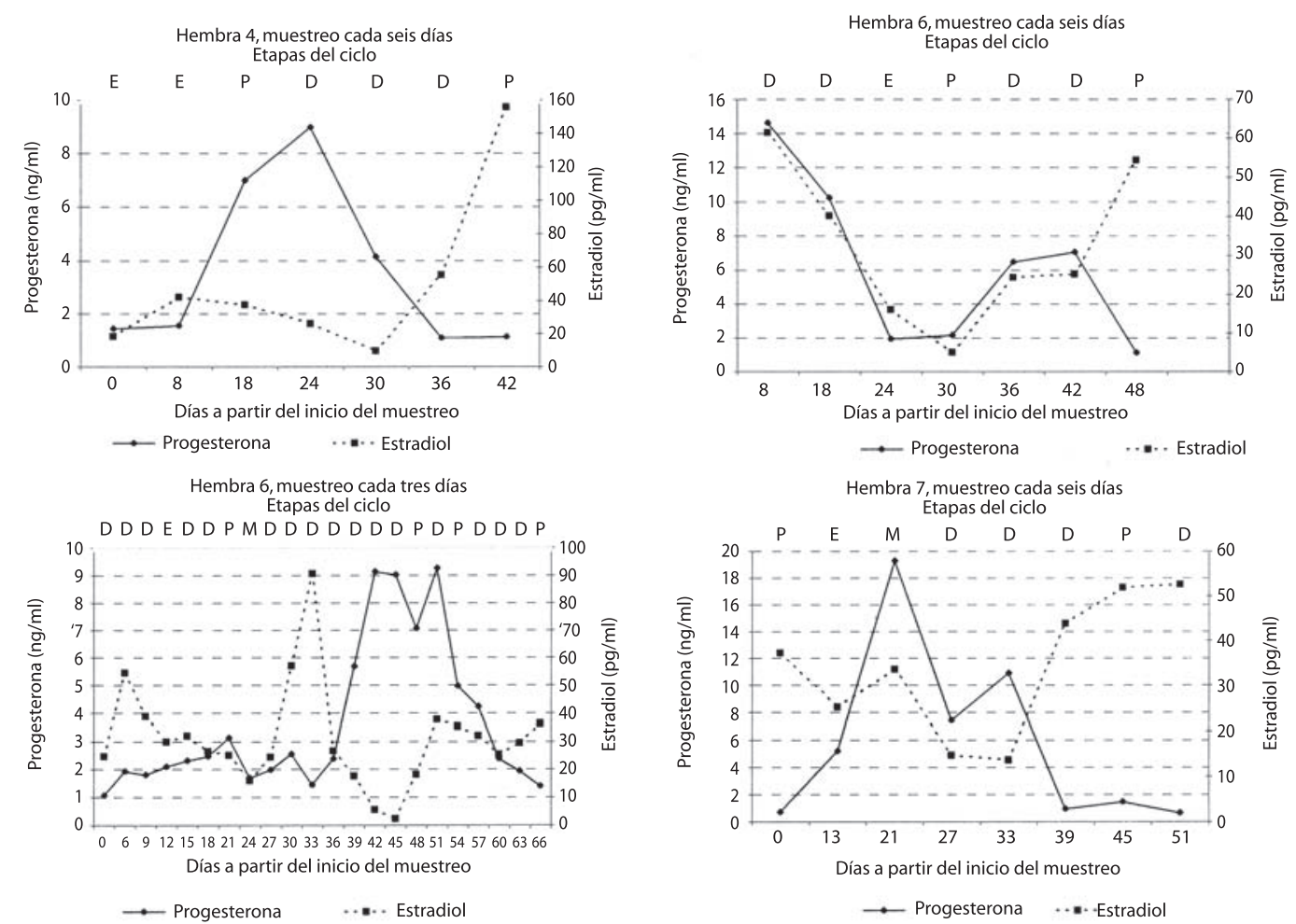

$\rightarrow$ Progesterona

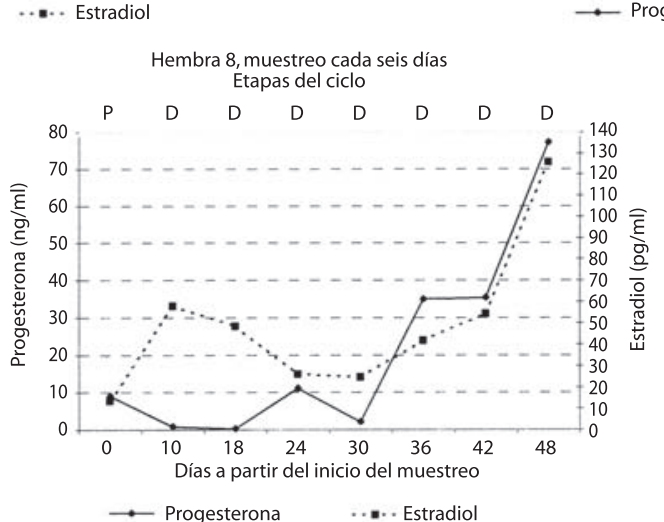

Fig 1. Niveles de progesterona sanguínea en nanogramos por un mililitro de plasma (ng/ml) y $17 \beta$ estradiol en picogramos por un mililitro (pg/ml) en tepezcuintles (Agouti paca) de las hembras 4, 6, 7 y 8 y su correspondiente etapa del ciclo en P (proestro), E (estro), M (metaestro), D (diestro).

Fig 1. Levels of blood progesterone in nanograms per mililiter of plasma (ng/ml) and $17 \beta$ estradiol in picograms per mililiter (pg/ml) in females of tepezcuintle (Agouti paca) 4, 6, 7 and 8 in her cycle phase P (proestrus), E (oestrus), M (metaoestrus), $\mathrm{D}$ (dioestrus).

P4 característico de un ambiente estrogénico, lo que indica una fase folicular, sin embargo, la hembra 4 presentó un cuerpo luteo principal (CLP). En ambos casos se observaron folículos en diferentes etapas de desarrollo. La hembra 1 presentó una proporción de P4 mayor que E2, indicativo de un ambiente progestágeno y por lo tanto de una fase luteal, lo cual va de acuerdo a la presencia de un CLP claramente definido macro y microscópicamente en el ovario 


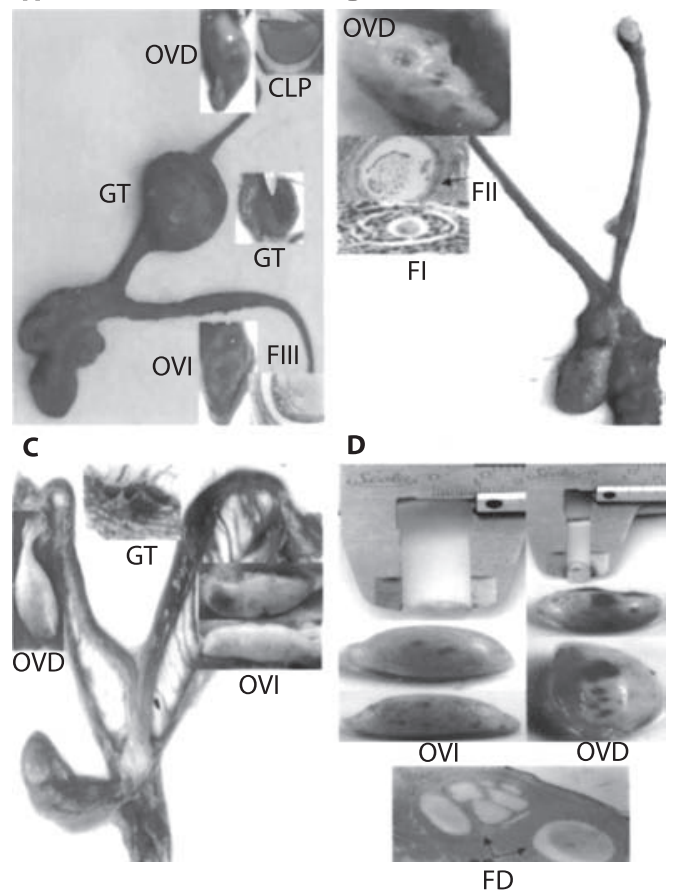

Fig 2. Estructuras ováricas de las tepezcuintles 1 (A), 2 (B), 3 (C) y 4 (D). Gestación temprana (GT), cuerpo luteo principal (CLP), ovario derecho (OVD), ovario izquierdo (OVI), folículos en desarrollo (FD): FI (primario), FII (secundario), FIII (terciario).

Fig 2. Ovarian structures in tepezcuintles 1 (A), 2 (B), 3 (C) y 4 (D). Early gestation (GT), corpus luteum (CLP), right ovary (OVD), left ovary (OVI), growth follicles (FD): FI (primary), FII (secondary), FIII (tertiary).

derecho (Fig. 2A). Se observó también la presencia de varios folículos en diferentes etapas de crecimiento, al momento de la necropsia se encontró un producto en útero.

La hembra 3 presentó una proporción relativamente alta de P4 y E2, mostrando un ambiente progestágeno congruente con una fase luteal por la presencia de un cuerpo luteo en el ovario izquierdo (Fig. 2C). En la hembra 7 endocrinológicamente se observó una fase luteal, no típica como en las hembras anteriores, debido a la presencia de numerosos folículos en diferentes etapas de desarrollo. En el Cuadro 3 se indican las características macro y microscópicas de las estructuras ováricas de cinco hembras con sus correspondientes niveles de P4 y E2.

\section{DISCUSIÓN}

Los niveles basales de P4 para machos castrados de A. paca son valores relativamente bajos, pero superiores a $0.0 \mathrm{ng} / \mathrm{ml}$; en hembras no gestantes también se encontraron niveles basales similares (entre 0.1 y $0.5 \mathrm{ng} / \mathrm{ml}$ ). En cuanto a los niveles basales de E2 en machos castrados son también superiores a $0.0 \mathrm{pg} / \mathrm{ml}$, así como para hembras no gestantes. Los niveles basales de esteroides circulantes en A. paca machos castrados son de origen extragonadal; se ha reportado que las fuentes extragonadales de P4 son la corteza adrenal y la placenta, mientras que para E2 son los adipocitos, el sistema nervioso central, corteza adrenal, músculo y placenta (Cupps 1991).

La presencia de P4 adrenal ha sido informado por Plotka et al. (1983) y Asher et al. (1989) en varias especies como los cérvidos y ratas. Zaneveld y Chatterton (1982) mencionan que los niveles de progesterona y estradiol adrenales en los mamíferos son bajos, apenas detectables; sin embargo, en A. paca, los niveles basales de estos esteroides son mayores. Especialmente los niveles elevados de E2 observados en A. paca pueden estar en función de una actividad de aromatasa elevada en los tejidos mencionados.

Bajo este contexto se puede pensar que los niveles hormonales estimados por RIA para $A$. paca, procedentes de gónada fueron sobreestimados, sin embargo, esto no se puede afirmar para todos los casos, pues aunque se observó una amplia variación individual se encontraron valores de E2 de 0 pg/ml en machos gonadectomizados y no gonadectomizados. Situación de esta naturaleza se presentó en un animal joven (hembra 2), la cual mostró fluctuaciones de 0 a $23 \mathrm{pg} / \mathrm{ml}$ con presencia de numerosos folículos en crecimiento, pero sin la presencia de un CL, lo que indicó incipiente actividad ovárica.

En la presente investigación se observó que la producción de esteroides extragonadales es constante y sin variaciones entre los 10 y 40 min posteriores al inicio de la sedación del animal, situación distinta se presenta en el venado cola blanca (Odocoileus virginianus), 
CUADRO 3

Características macroscópicas y microscópicas de los ovarios de cinco tepezcuintles (Agouti paca)

TABLE 3

Macroscopic and microscopic ovarian characteristics of five tepezcuintles (Agouti paca)

Niv. Hor.

Iden

$\begin{array}{lll}01 & 23.00 & 10.02\end{array}$

Di

D, I CLP rojizo ipsilateral,

2 Folículos

Cuerno derecho con un

Producto de $1 \mathrm{~cm}$ de diámetro

Gestación temprana

I Folículos de $1 \mathrm{~mm}$ de diámetro

D 13 Folículos de $1 \mathrm{~mm}$ de diámetro

I 10 Folículos de $1 \mathrm{~mm}$ de diámetro

$\begin{array}{llll}03 & 37.36 & 68.26 & \mathrm{~N}\end{array}$

D Ovario estático

$(1.03 \times 0.5 \mathrm{~cm})$

I 1 Folículo de $1 \mathrm{~mm}$ de diámetro (ovario $1.1 \times 0.56 \mathrm{~cm}$ ) 1 CL de $1 \mathrm{~mm}$ de diámetro aproximadamente Gestación temprana

$\begin{array}{llll}04 & 1.13 & 155.84 & \mathrm{P}\end{array}$

$1 \mathrm{~mm}$ de

diámetro

(ovario $1.46 \times 1.09 \mathrm{~cm}$ )

I 16 Folículos de $1 \mathrm{~mm}$ de diámetro

1 Folículo de $1.5 \mathrm{~mm}$ de diámetro

1 Folículo de $2 \mathrm{~mm}$ de diámetro

(ovario $1.2 \times 0.7 \mathrm{~cm}$ )

Microscópicas (mm)

\begin{tabular}{|c|c|c|c|c|}
\hline \multirow[t]{2}{*}{ F I } & F II & F III & CLP & Otros \\
\hline & $\begin{array}{c}2 \\
4 \mathrm{x} \\
1.76 \times 1.58 \\
\text { Antro } \\
1.14 \times 1.17 \\
10 \mathrm{x} \\
\text { T.E. } 0.07 \\
\text { T.I. } 0.05\end{array}$ & $\begin{array}{c}1 \\
4 \mathrm{x} \\
1.61 \times 2.19 \\
\text { Antro } \\
1.70 \times 1.17 \\
10 \mathrm{x} \\
\text { T.E. } 0.11 \\
\text { T.I. } 0.10\end{array}$ & $\begin{array}{c}1 \\
4 \mathrm{x} \\
4.9 \times 5.19\end{array}$ & 3 Folículos atrésicos \\
\hline 1 & 1 & 1 & & 1 Ovocito \\
\hline $4 \mathrm{x}$ & $4 \mathrm{x}$ & $4 \mathrm{x}$ & & $10 \mathrm{x}$ \\
\hline $1.29 \times 0.99$ & $\begin{array}{c}1.41 \times 1.05 \\
\text { Antro } \\
0.41 \times 0.67 \\
10 \mathrm{x} \\
\text { T.E. } 0.09 \\
\text { T.I. } 0.11\end{array}$ & $\begin{array}{l}2.49 \times 2.40 \\
\quad \text { Antro } \\
2.07 \times 1.64 \\
\text { T.E. } 0.23 \\
\text { T.I. } 0.17\end{array}$ & & $\begin{array}{c}0.11 \times 0.19 \\
1 \text { Foliculo atrésico } \\
4 \times \\
1.29 \times 1.05\end{array}$ \\
\hline 3 & $\begin{array}{c}2 \\
10 \mathrm{x} \\
1.01 \times 0.89 \\
\text { Antro } \\
0.83 \times 0.54 \\
\text { T.E. } 0.11 \\
\text { T.I. } 0.05\end{array}$ & & & $\begin{array}{c}1 \text { Folículo atrésico } \\
10 \mathrm{x} \\
0.83 \times 0.74\end{array}$ \\
\hline & $\begin{array}{c}2 \\
4 \mathrm{x} \\
0.99 \times 0.85\end{array}$ & & & $\begin{array}{c}1 \text { Folículo atrésico } \\
10 \mathrm{x} \\
0.21 \mathrm{x} 0.16\end{array}$ \\
\hline & & 1 & 1 & \\
\hline 1 & 2 & & $\begin{array}{c}1 \\
4 \mathrm{x} \\
3.26 \times 2.94 \\
\text { T.E. } 0.11 \\
\text { T.I. } 0.08\end{array}$ & $\begin{array}{c}1 \text { Folículo atrésico } \\
1 \text { Ovocito } \\
4 \mathrm{x} \\
0.52 \times 0.47\end{array}$ \\
\hline & 2 & & & $\begin{array}{c}1 \text { Cuerpo lúteo } \\
\text { accesorio } \\
10 \mathrm{x} \\
0.23 \times 0.27 \\
1 \text { Ovocito } \\
10 \mathrm{x} \\
0.09 \times 0.07\end{array}$ \\
\hline
\end{tabular}


CUADRO 3 (Continuación)

Características macroscópicas y microscópicas de los ovarios de cinco tepezcuintles (Agouti paca)

TABLE 3 (Continued)

Macroscopic and microscopic ovarian characteristics of five tepezcuintles (Agouti paca)

Niv. Hor.

$\begin{array}{crrrrr}\text { Ident. } & \text { P4 } & \text { E2 } & \text { CV } & \text { OV } & \\ 07 & 4.28 & 68.05 & \mathrm{~N} & \text { D } & \begin{array}{l}3 \text { Folículos rojizos de } 1 \mathrm{~mm} \\ \text { de diámetro } \\ \end{array} \\ & & & \begin{array}{l}1 \text { Folículo rojizo de } 0.5 \mathrm{~mm} \\ \text { de diámetro }\end{array}\end{array}$

I 3 Folículos rojizos de $1 \mathrm{~mm}$ de diámetro

2 Folículos rojizos de $0.5 \mathrm{~mm}$ de diámetro
Microscópicas (mm)

\begin{tabular}{|c|c|c|c|c|}
\hline F I & F II & F III & CLP & Otros \\
\hline 1 & \multirow[t]{8}{*}{3} & 1 & & \\
\hline $10 \mathrm{x}$ & & $4 \mathrm{x}$ & & \\
\hline $0.95 \times 0.86$ & & $2.82 \times 2.46$ & & \\
\hline T.E. 0.13 & & Antro & & \\
\hline \multirow[t]{12}{*}{ T.I. 0.15} & & $2.07 \times 2.40$ & & \\
\hline & & $10 \mathrm{x}$ & & \\
\hline & & T.E. 0.14 & & \\
\hline & & T.I. 0.03 & & \\
\hline & \multirow[t]{8}{*}{1} & 1 & & 1 Ovocito \\
\hline & & $4 \mathrm{x}$ & & $10 \mathrm{x}$ \\
\hline & & $2.13 \times 2.43$ & & $0.35 \times 0.21$ \\
\hline & & Antro & & \\
\hline & & $1.70 \times 1.55$ & & \\
\hline & & $10 \mathrm{x}$ & & \\
\hline & & T.E. 0.08 & & \\
\hline & & T.I. 0.03 & & \\
\hline
\end{tabular}

Niv. Hor: Niveles Hormonales

I: Ovario izquierdo

CLP: Cuerpo luteo principal

P: Proestro

P4: progesterona en $\mathrm{ng} / \mathrm{ml}$

\author{
Ident.: Identificación \\ FI: Folículo I \\ T.E: Teca externa \\ $\mathrm{N}$ : Sin muestra citológica \\ E2: 17 beta estradiol (pg/ml)
}

OV: Ovario
FII: Folículo II
T.I.: Teca interna
CV: citología vaginal
4x: cuatro aumentos

una misma muestra con estuches comerciales diferentes tienen variaciones que son atribuibles a las características de los calibradores correspondientes a cada estuche comercial (Epelu-Opio y Madej 1988), esto es en razón de que Guimarâes et al. (1999) utililizaron un estuche comercial diferente (Coat-A-Count, Diagnostic Products, Co) al del presente trabajo. Adicionalmente Guimarâes et al. (1999) mencionan que encontraron niveles más altos en diestro respecto a proestro, estro y metaestro pero no mencionan si existen diferencias significativas entre estos niveles.

Se encontraron niveles altos de P4 (de 20 a $35.4 \mathrm{ng} / \mathrm{ml}$ ) durante el ciclo estral comparado con otras especies del mismo suborden. La razón de esto podría ser una estrategia reproductiva de A. paca para brindar las condiciones uterinas apropiadas para la gestación, ya que 
ésta se extiende por un período de tiempo relativamente largo (Weir y Rowlands 1974). Entre los autores que reportan niveles de P4 en otros histricomorfos se encuentran: Rowlands y Heap (1966), quienes registran niveles entre 2 y $6 \mathrm{ng} / \mathrm{ml}$ para Myocastor coypu; Tam (1974) informó entre 8 y 18 ng/ml para Galea musteloides y Guimarâes et al. (1997), mencionan niveles entre 0.22 y $9.02 \mathrm{ng} / \mathrm{ml}$ para Dasyprocta primnolopha.

Para el muestreo de cada seis días, en la hembra 6, se observaron altos niveles de E2, paralelos a los de P4 durante la fase luteal. Estos altos niveles probablemente provienen en su mayor proporción del tejido ovárico. Tal afirmación está fundamentada en los hallazgos histológicos de la hembra 7, la cual mostró altos valores de esteroides en presencia de diversas estructuras foliculares y tejido intersticial pero sin la existencia de un CLP, posiblemente debido a que algunos folículos presentes pudiesen estar en alguna fase de luteinización y es por eso que los niveles de P4 y E2 se encontraron relativamente altos. Esta situación indicó que el animal cursaba por una fase luteal, similar a los perfiles hormonales de las hembras 1 y 3 que gestaron.

Los hallazgos descritos apoyan la hipótesis de Weir y Rowlands (1974), en cuanto a que el crecimiento folicular está destinado a proveer tejido intersticial con capacidad progestágena, ya sea por la atresia folicular o por la formación de cuerpos lúteos accesorios (CLA), con el propósito de crear un ambiente endocrino propicio para iniciar y mantener una gestación larga. En el caso de $A$. paca, ésta es una estrategia adaptativa de la reproducción, para lograr la formación de crías maduras al nacimiento, que tendrían una probabilidad mayor de supervivencia respecto a otras especies de roedores.

En las hembras 1 y 3 se apreció un ambiente progestágeno con presencia de un CLP y producto en útero, además folículos en crecimiento. En las hembras 2 y 4 se observó un ambiente estrogénico con presencia de estructuras foliculares; sin embargo, presentaron niveles de P4 de 1.33 y 1.13 ng/ml respectivamente, que son relativamente altos comparados con otras especies domésticas (búfalo, vaca, oveja y cabra), en las cuales se asume que valores iguales o mayores de $1 \mathrm{ng} / \mathrm{ml}$ indican actividad luteal (Dobson 1983). Es probable que las cantidades basales de P4 mostradas por las hembras 2 y 4, provengan del mismo ovario pero no de CLP. Dicha afirmación está fundamentada en lo propuesto por Weir y Rowlands (1974), las cuales mencionan que el ovario de A. paca, Dasyprocta agouti, Chinchilla laniger e Hystrix africaeaustralis presentan rasgos diferentes a las especies domésticas, caracterizado por abundante tejido intersticial, CLA y folículos atrésicos, todos con capacidad esteroidogénica, cuyas capacidades de producción de hormonas esteroides son más altas que en otras especies. También cabe la posibilidad de que estos valores sean aporte de las fuentes extragonadales, aunque no se pudo precisar en el presente informe el aporte de cada fuente, las variaciones fluctuaron entre 0.8 y $3.7 \mathrm{ng} / \mathrm{ml}$ para P4 y de 0 y 73 pg/ml para E2.

Mossman y Judas citados por Weir y Rowlands (1974), mencionan que las células de la teca interna de Hystrix cristata tienen carácter pluripotencial, se asume que esta característica se extiende a otros histricomorfos. Los resultados del presente estudio aportan datos que apoyan este argumento para $A$. paca, en virtud de que las observaciones al microscopio de los cortes histológicos mostraron grupos de células luteinizadas producto de folículos atrésicos y/o tejido intersticial.

En general, los niveles de E2 mostraron amplia variabilidad, esto puede estar relacionado con la idiosincrasia individual de las hembras y a un crecimiento folicular constante que aporta por diferentes vías cantidades variables de E2, teniendo como posibles intermediarios la $20 \alpha-$ hidroxi-4-pregnen-3-ona, $17 \alpha$-hidroxipregnenolona y progesterona, que provienen según Tam (1974) del tejido intersticial ovárico y folículos. Los altos valores presentes de E2 en la fase folicular y los bajos niveles de P4 en la misma fase indican que probablemente el principal precursor no es la P4, si no la pregnenolona que sigue la vía $\Delta 5$, tal como lo reporta Tam (1974) para Chinchilla laninger y Cavia porcellus. 
Se concluye que hay producción de progesterona y $17 \beta$-estradiol extragonadal en tepezcuintles machos gonadectomizados. Existen diferencias en las cantidades de progesterona sanguínea entre la fase luteal y folicular del ciclo estral pero no así para estradiol y, el ciclo ovárico dura $29 \pm 8.4$ días.

\section{AGRADECIMIENTOS}

Los autores agradecen profundamente el financiamiento otorgado por el Consejo Nacional de Ciencia y Tecnología (CONACYT) mediante el convenio 489100-5-0294PB y a la Comisión Nacional para el Conocimiento y Uso de la Biodiversidad (CONABIO), convenio FB410/ M005/97 para efectuar el presente estudio.

\section{RESUMEN}

Se caracterizó la actividad ovárica de A. paca por medio de perfiles hormonales y estructuras ováricas. Se muestrearon ocho hembras (siete adultas y una juvenil) en el criadero de la Facultad de Medicina Veterinaria y Zootecnia en el estado de Yucatán, México, durante aproximadamente dos meses. Se recolectaron muestras sanguíneas cada 3 y 6 días en animales anestesiados. Se estimaron los niveles de progesterona (P4) y $17 \beta$ estradiol (E2) sanguíneos por radioinmunoanálisis. Las estructuras ováricas de animales muertos durante el periodo de muestreo fueron analizadas macro y microscópicamente. El ciclo ovárico duró $29 \pm 8.4$ días, con niveles de $1.61 \pm 0.65 \mathrm{ng} / \mathrm{ml}$ para P4 y de $39 \pm 24 \mathrm{pg} / \mathrm{ml}$ para E2 durante la fase folicular, y de $6.18 \pm 3.70 \mathrm{ng} / \mathrm{ml}$ y $29 \pm 16 \mathrm{pg} / \mathrm{ml}$ para P4 y E2 respectivamente, en la fase luteal. Hubo diferencias $(\mathrm{p}<0.05)$ en los niveles de $\mathrm{P} 4$ entre las fases folicular y luteal, no así para E2. Se detectó la presencia de esteroides extragonadales, con niveles de $1.9 \pm 0.77 \mathrm{ng} /$ $\mathrm{ml}$ para $\mathrm{P} 4$ y de $22 \pm 17 \mathrm{pg} / \mathrm{ml}$ para E2, los cuales no son secretados por efecto del estrés por manejo. Los cambios en los niveles de P4 durante el ciclo son indicadores de actividad luteal, funcionando el tejido intersticial probablemente como una glándula productora de esteroides. De igual forma se observó que el crecimiento folicular ocurre durante todo el ciclo.

Palabras clave: Agouti paca, esteroides, ciclo estral, progesterona, estradiol, actividad ovárica, histricomorfo.

\section{REFERENCIAS}

Anónimo 1984. Laboratory training manual on radioimmunoassay in animal reproduction. Technical Reports Series No. 233. International Atomic Energy Agency, Viena. 269 p.

Asher, G.W., A.J. Peterson \& D. Duganzich. 1989. Adrenal and ovarian sources of progesterone secretion in young female fallow deer, Dama dama. J. Reprod. Fert. 85: 667-675.

Cabrera, B.E.A. \& R.C. Montes. 1999. Estudio de la actividad ovárica de tepezcuintles (Agouti paca) mediante los niveles de progesterona sanguinea y estradiol plasmáticos. XVI Congreso de la Asociación de Zoológicos, Criaderos y Acuarios de la República Mexicana. Guadalajara, Jalisco, México.

Cupps, P.T. 1991. Reproduction in domestic animals. Academic, Nueva York, Nueva York, EEUU. 670 p.

Dobson, H. 1983. A radioimmunoassay laboratory handbook. Liverpool University, Liverpool, Gran Bretaña. $91 \mathrm{p}$.

Downie, N.M. \& R.W. Heath. 1986. Métodos estadísticos aplicados. Harla, México D.F., México. 380 p.

Duch, G.J. 1988. La conformación territorial del Estado de Yucatán, México. Universidad Autónoma de Chapingo. Estado de México, México. 428 p.

Epelu-Opio, J. \& A. Madej.1988. Direct measurement of progesterone in plasma and milk by a simple solidphase radioimmunoassay. Vet. Clin. Path. 7: 25-28.

Fierro, S.M. \& L.M.S. Morales. 1995. Caracterización del ciclo estral en el tepezcuintle (Agouti paca) por medio de frotis vaginales. Tesis de licenciatura. Facultad de Estudios Profesionales Cuautitlán. Universidad Nacional Autónoma de México, Cuautitlán Izcalli, Estado de México, México. 45 p.

García, E. 1988. Modificaciones al sistema de clasificación climático de Köppen. Cuarta edición. Instituto de Geografía. Universidad Nacional Autónoma de México, México, D.F. 213 p.

Guimarâes, D.A., O.M. Ohashi \& W.G. Vale. 1997. Níveis de progesterona plasmática durante o ciclo estral de cutia (Dasyprocta prymnolopha) criadas em cativeiro. III Congreso Internacional sobre Manejo de Fauna Silvestre en Amazonia. Santa Cruz, Bolivia. 45 p. 
Guimarâes, D.A., A.P. Lameira \& O. Ohashi. 1999. Perfil hormonal de progesterona no ciclo estral da Agouti paca -estudo preliminar. IV Congreso Internacional sobre Manejo de Fauna Silvestre en Amazonia y Latinoamérica. Asunción, Paraguay: 117-118.

Matamoros, Y. \& B. Pashov. 1984. Ciclo estral del tepezcuinte (Cuniculus paca, Brisson), en cautiverio. Brenesia 22: 249-260.

Montes, P.R.C. 1995. Los ensayos de unión para medir hormonas. Rev. Biomed. 6: 33-46.

Plotka, E.D., U.S. Seal, L.J. Verme \& J.J. Ozoga. 1983. The adrenal gland in white-tailed deer: A significance source of progesterone. J. Wild. Manage. 47: 38-44.

Rowlands, I.W. \& R.B. Heap. 1966. Histological observations on the ovary and progesterone levels in the coypu (Myocastor соурu). Symp. Zool. Soc. Lond. 15: 335-355.
Sanvicente, M.L. 1995. El tepezcuintle (Cuniculus paca) como alternativa de proteína animal para consumo humano en el trópico húmedo estudio recapitulativo. Tesis de licenciatura. Facultad de Medicina Veterinaria y Zootecnia. UNAM. México, D.F. 120 p.

Smythe, N. \& O. Brown de Guanti. 1995. La domesticación y cría de la paca (Agouti paca). Guía de conservación No. 26. FAO, Roma. 91 p.

Tam, W.H. 1974. Synthesis of progesterone in some hystricomorph rodents. Symp. Zool. Soc. Lond. 64: 363-384.

Weir, B.J. \& I.W. Rowlands. 1974. Functional anatomy of histricomorph ovary. Symp. Zool. Soc. Lond. 34: 303-332.

Zaneveld, L.J.D. \& R.T. Chatterton. 1982. Biochemistry of mammalian reproduction. John Wiley, Nueva York, Nueva York, EEUU. 561 p. 